\title{
Feasibility of Single-Incision Plus One Port Laparoscopic Low Anterior Resection for Rectal Cancer
}

\author{
Jae Young Kwak, M.D., Ph.D., Kwan Mo Yang, M.D., Myeong Sik Han, M.D., Ph.D. \\ Department of Surgery, Gangneung Asan Hospital, University of Ulsan College of Medicine, Gangneung, Korea
}

Purpose: Single-incision laparoscopic surgery is a recently developed minimally invasive surgical technique. We aimed to compare the feasibility and safety of single-incision plus one port laparoscopic low anterior resection (S+1-LAR) with those of multi-port laparoscopic low anterior resection (M-LAR) for mid-to-low rectal cancer.

Methods: We retrospectively reviewed patient characteristics and surgical outcomes by assessing data collected from the medical records of patients who underwent elective laparoscopic low anterior resection for mid-to-low rectal cancer at the Gangneung Asan Hospital.

Results: From April 2015 to April 2019, 52 patients underwent S+1-LAR ( $\mathrm{n}=28)$ or M-LAR ( $\mathrm{n}=24)$ for midto-low rectal cancer at Gangneung Asan Hospital. There were no significant between-group differences in clinical characteristics. The mean postoperative 1-day pain score was significantly lower in the S+1-LAR group. Surgical outcomes and postoperative complications did not differ significantly between the two groups.

Conclusion: S+1-LAR is a feasible and safe technique and is comparable with M-LAR in terms of surgical outcomes of patients with mid-to-low rectal cancer.

Keywords: Single incision, Rectal cancer, Laparoscopic surgery, Complication, Minimally invasive surgery

This is an Open Access article distributed under the terms of the Creative Commons Attribution Non-Commercial License (http:// creativecommons.org/licenses/by-nc/4.0/) which permits unrestricted non-commercial use, distribution, and reproduction in any medium, provided the original work is properly cited.

\author{
Received May 11, 2020 \\ Revised 1st June 27, 2020 \\ 2nd July 31,2020 \\ Accepted August 26, 2020 \\ Corresponding author \\ Myeong Sik Han \\ Department of Surgery, Gangneung \\ Asan Hospital, University of Ulsan \\ College of Medicine, 38 Bangdong-gil, \\ Sacheon-myeon, Gangneung 25440, \\ Korea \\ Tel: +82-33-610-3218 \\ Fax: +82-33-641-8120 \\ E-mail: mshan777@hotmail.com \\ ORCID: \\ https://orcid.org/0000-0001-6918-4432
}

Copyright (๐) 2020 The Journal of Minimally Invasive Surgery. All rights reserved.

\section{INTRODUCTION}

There are several surgical treatment options for colorectal cancer, including the recently developed single incision laparoscopic approach. The safety and feasibility of this intervention for colorectal cancer have been reported by many surgeons. ${ }^{1.5}$ However, single incision laparoscopic low anterior resection for rectal cancer is technically difficult because of the limited instrument maneuverability, loss of triangulation, and limited in-line visualization. ${ }^{6}$ Single-incision plus one port laparoscopic low anterior resection (S+1-LAR) helps overcome these technical difficulties, particularly in the rectal division, when treating mid-to-low rectal cancer. ${ }^{7-10}$ However, S+1-LAR has not been assessed in comparison with multi-port laparoscopic low anterior resection (M-
LAR).

Therefore, this study aimed to compare the safety and feasibility of S+1-LAR and M-LAR for mid-to-low rectal cancer.

\section{MATERIALS AND METHODS}

Between April 2015 and April 2019, 233 patients underwent lower anterior resection for rectal cancer at Gangneung Asan Hospital, Gangneung, Korea. Patients were included if they had (1) a histologically proven adenocarcinoma, (2) tumors located in the mid-to-low rectum, or (3) undergone laparoscopic low anterior resection. We excluded patients who underwent combined operation (ex. cholecystectomy, hysterectomy etc.) and distant metastasis. Patients' clinical characteristics, surgical outcomes, 
postoperative complications, pathologic features, and oncologic outcomes were compared between those who underwent S+1LAR and those who underwent M-LAR.

For bowel preparation, a polyethylene glycol electrolyte solution was administered the day before surgery. A second-generation cephalosporin was administered intravenously $30 \mathrm{~min}$ before surgery.

All operations were performed by two experienced colorectal surgeons. All patients were prepared in the lithotomy position with general anesthesia. For patients in the S+1-LAR group, a 4-cm vertical incision was made through the umbilicus of 25 patients and the right side of the abdomen in view of raising an ileostomy of 3 patients, and a single port $\left(\right.$ GelPOINT $^{\circledR}$, Applied Medical, Rancho Santa Margarita, CA, USA) was inserted. One $12-\mathrm{mm}$ additional port was placed in the right lower quadrant of the abdomen. For those in the M-LAR group, five ports were used, including a camera port. Five-millimeter ports were placed in the left upper, left lower, and right upper abdominal quadrants. Twelve-millimeter ports were placed through the umbilicus and right lower quadrant (Fig. 1). The rectum and sigmoid colon were mobilized using a medial-to-lateral approach. Mobilization of the splenic flexure was not performed routinely except in patients with a lack of redundancy in the sigmoid colon. The distal rectum was dissected by inserting an endoscopic linear stapling device through the right lower channel in both groups. Resection was achieved following extracorporealization, and anastomosis was performed with the double stapling technique using a trans-anally inserted circular stapler. A pelvic drainage tube was inserted from the left lower abdominal quadrant. Only standard straight laparoscopic instruments were used.

Sips of water were allowed on the third postoperative day, and liquid and soft diet was allowed after flatus. The Foley catheter was removed on the third postoperative day. Patient-controlled opioid-based intravenous analgesia (PCIA) was used routinely on postoperative days 1 and 2 and additional analgesics were administered when the patient complained of severe pain. The patients were discharged if they could tolerate a soft diet and ambulate independently.

Postoperative pain was recorded using the numeric rating scale (NRS) daily until the patient was discharged. The patient was asked to make three pain ratings, corresponding to current, best and worst pain experienced over the past 24 hours on a scale of 0 (no pain) to 10 (worst pain imaginable). The average of the 3 ratings was used to represent the patient's level of pain in the 24 hour period.

Categorical variables were analyzed using the $\chi^{2}$ test, whereas continuous variables were analyzed using the Student's t test or Fisher's exact test. All tests were 2-sided. A $p$ value less than 0.05 was considered statistically significant. All data were analyzed using the PASW Statistics for Windows, Version 18.0 (SPSS Inc., Chicago, IL, USA).

We performed a retrospective cross-sectional analysis. Medical records, surgical findings, pathology reports, and radiologic reports were reviewed. The primary outcomes were postoperative pain and overall postoperative complication, defined as all postoperative complications which occurred on the day after surgery. The secondary outcomes were operating time, rate of conversion to open, quality of specimen, port site hernia rate (within the first year after surgery). The Institutional Review Board of GNAH approved this study and waived the need to obtain informed consent (GNAH 2019-11-014).

\section{RESULTS}

Fifty-two patients with a mean age of 67.5 years underwent laparoscopic low anterior resection during the study period. S+1LAR was performed in 28 patients and M-LAR was performed in 24 patients. The mean follow-up time was 23.3 months, and the median follow-up period was 26.5 and 19.6 months in the S+1LAR and M-LAR groups, respectively. Preoperative chemoradiotherapy was administered significantly more frequently in the M-LAR group than in the S+1-LAR group $(p=0.040)$. Otherwise, there were no significant differences between the two groups in terms of age, gender, body mass index, preoperative carcinoem-
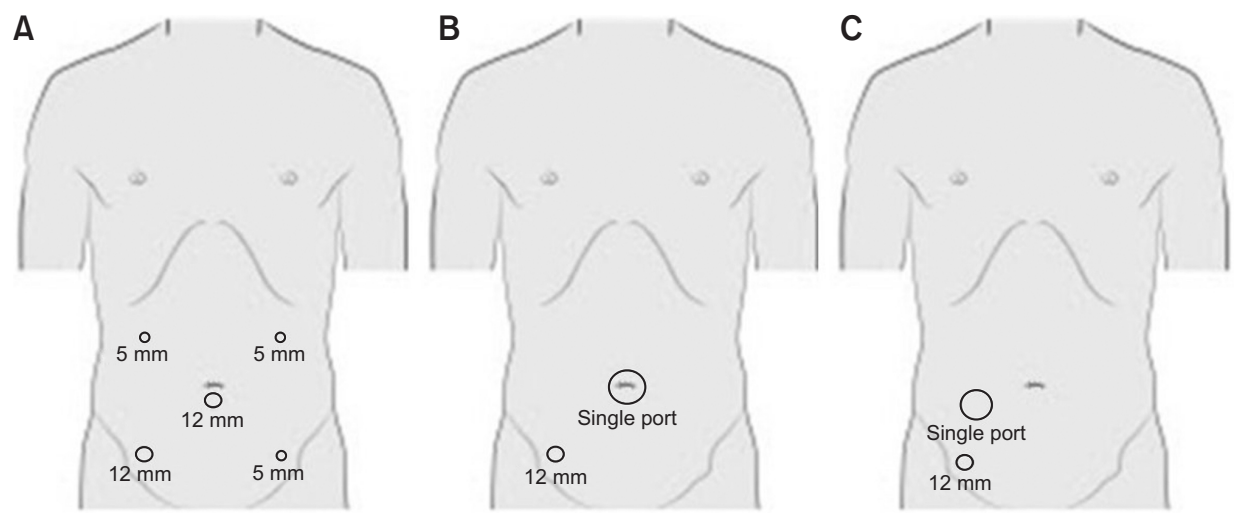

Fig. 1. Port placement for patient who underwent multi-port laparoscopic low anterior resection (M-LAR) $(A)$, single incision plus one port laparoscopic low anterior resection (S+1-LAR) using umbilicus as main incision (B) and single incision plus one port laparoscopic low anterior resection (S+1-LAR) using ileostomy site as main incision (C). 
Table 1. Patient clinical characteristics

\begin{tabular}{|c|c|c|c|}
\hline & $\mathrm{S}+1-\mathrm{LAR}(\mathrm{n}=28)$ & $M-L A R(n=24)$ & $p$ value \\
\hline Mean age, years (range) & 68.4 (48 82) & $66.4(44 \sim 83)$ & 0.492 \\
\hline Male & $22(78.5)$ & $17(70.8)$ & 0.541 \\
\hline $\mathrm{BMI}, \mathrm{kg} / \mathrm{m}^{2}$ & $23.8 \pm 3.8$ & $25.2 \pm 3.3$ & 0.158 \\
\hline Preoperative CEA (ng/ml) & $3.5 \pm 2.3$ & $6.5 \pm 13.1$ & 0.239 \\
\hline \multicolumn{4}{|l|}{ Comorbidity } \\
\hline Hypertension & $13(46.4)$ & $18(75.0)$ & 0.049 \\
\hline Diabetes mellitus & $6(21.4)$ & $5(20.8)$ & 1.000 \\
\hline Tuberculosis & $3(10.7)$ & $2(8.3)$ & 1.000 \\
\hline Cerebrovascular disease & $1(3.6)$ & $1(4.2)$ & 1.000 \\
\hline COPD & $3(10.7)$ & $1(4.2)$ & 0.615 \\
\hline Ischemic heart disease & $5(17.9)$ & $4(16.7)$ & 1.000 \\
\hline Prior abdominal surgery & $6(21.4)$ & $4(16.7)$ & 0.736 \\
\hline Preoperative CRT & $1(3.6)$ & $6(25.0)$ & 0.040 \\
\hline Postoperative CRT & $4(14.3)$ & $2(8.3)$ & 0.674 \\
\hline
\end{tabular}

Values are presented as number (\%) or mean \pm standard deviation unless otherwise stated. S+1-LAR = single-incision plus one port laparoscopic low anterior resection; $\mathrm{M}$-LAR = multi-port laparoscopic low anterior resection; $\mathrm{BMl}=$ body mass index; $\mathrm{CEA}=$ carcinoembryonic antigen; COPD = chronic obstructive pulmonary disease; CRT = chemoradiotherapy.

Table 2. Surgical procedure details

\begin{tabular}{|c|c|c|c|}
\hline & $\mathrm{S}+1-L A R(\mathrm{n}=28)$ & $M-L A R(n=24)$ & $p$ value \\
\hline Mean operative time, min (range) & $196.9(115 \sim 265)$ & $210.8(120 \sim 345)$ & 0.298 \\
\hline Tumor location from anal verge, cm & $8.4 \pm 2.1$ & $7.3 \pm 2.3$ & 0.77 \\
\hline Mean anastomosis level from anal verge, cm (range) & $5.7(2 \sim 10)$ & $4.7(2 \sim 9)$ & 0.085 \\
\hline Protective diverting ileostomy & $20(71.4)$ & $19(79.1)$ & 0.749 \\
\hline Conversion to open surgery & 0 & $1(4.2)$ & 0.462 \\
\hline Conversion to multi-port surgery & 0 & - & - \\
\hline Using ileostomy site for main incision & 3 & 0 & 0.240 \\
\hline
\end{tabular}

Values are presented as number (\%) or mean \pm standard deviation unless otherwise stated. S+1-LAR = single-incision plus one port laparoscopic low anterior resection; M-LAR = multi-port laparoscopic low anterior resection.

bryonic antigen level, comorbidity, prior abdominal surgery, and postoperative chemoradiotherapy administration (Table 1).

Mean operative time and mean anastomosis level in the S+1LAR group were 196.9 minutes and $5.7 \mathrm{~cm}$ from anal verge, respectively. There were no significant differences between the two groups with regard to operative time, tumor location from the anal verge, anastomosis level, or frequency of diverting ileostomy creation. One patient in the M-LAR group was converted to open surgery because of severe adhesions. None of the patients in the S+1-LAR group required a switch to M-LAR (Table 2).

The mean postoperative 1-day pain score was significantly lower in the S+1-LAR group than in the M-LAR group ( $p=0.006)$.
Otherwise, the mean postoperative 3- and 7-day pain scores; interval to first flatus; and incidence of anastomosis leakage, anastomosis stricture, ileus, urinary complications, wound infection, and incisional hernia, did not differ significantly between the two groups. No perioperative deaths occurred in either group (Table 3).

The mean number of harvested lymph nodes and the mean distal resection margin in the S+1-LAR group were 15.6 and 2.6 $\mathrm{mm}$, respectively. There were no significant differences between groups in terms of tumor size, number of harvested lymph nodes, proximal margin, distal margin, tumor differentiation, tumor invasion, lymph node metastasis, lymphovascular inva- 
Table 3. Postoperative clinical outcomes and complications

\begin{tabular}{|c|c|c|c|}
\hline & $\mathrm{S}+1-\mathrm{LAR}(\mathrm{n}=28)$ & M-LAR $(n=24)$ & $p$ value \\
\hline \multicolumn{4}{|l|}{ Postoperative pain score, mean } \\
\hline 1 day & 3.64 & 4.75 & 0.006 \\
\hline 3 day & 2.71 & 3.04 & 0.396 \\
\hline 7 day & 1.86 & 2.00 & 0.243 \\
\hline Postoperative interval to first flatus, days & $2.79 \pm 1.2$ & $2.75 \pm 1.2$ & 0.915 \\
\hline Anastomosis leakage & 0 & 0 & - \\
\hline Anastomosis stricture & 2 & 1 & 1.000 \\
\hline lleus & 2 & 4 & 0.397 \\
\hline Urinary complication & 0 & 0 & - \\
\hline Wound infection & 2 & 0 & 0.493 \\
\hline Incisional hernia & 2 & 1 & 1.000 \\
\hline
\end{tabular}

Values are presented as number (\%) or mean \pm standard deviation unless otherwise stated. S+1-LAR = single-incision plus one port laparoscopic low anterior resection; M-LAR = multi-port laparoscopic low anterior resection.

Table 4. Pathologic features

\begin{tabular}{|c|c|c|c|}
\hline & $\mathrm{S}+1-\mathrm{LAR}(\mathrm{n}=28)$ & M-LAR $(n=24)$ & $p$ value \\
\hline Tumor size, cm (range) & $3.6 \pm 1.9(1.0 \sim 8.5)$ & $3.1 \pm 1.8(0.5 \sim 7.5)$ & 0.301 \\
\hline Number of harvested lymph nodes (range) & $15.6 \pm 8.7(4 \sim 50)$ & $13.1 \pm 4.8(4 \sim 22)$ & 0.223 \\
\hline Proximal resection margin, cm (range) & $10.1 \pm 4.9(2.6 \sim 23.0)$ & $8.8 \pm 4.5(2.0 \sim 21.0)$ & 0.325 \\
\hline Distal resection margin, cm (range) & $2.6 \pm 1.7(0.5 \sim 8.0)$ & $2.2 \pm 1.6(0.7 \sim 8.0)$ & 0.460 \\
\hline \multicolumn{4}{|l|}{ Tumor differentiation } \\
\hline WD & $9(32.1)$ & $5(20.8)$ & 0.761 \\
\hline $\mathrm{MD}$ & $18(64.3)$ & $18(75.0)$ & \\
\hline$P D+U D+M U C$ & $1(3.6)$ & $1(4.2)$ & \\
\hline \multicolumn{4}{|l|}{ Tumor invasion } \\
\hline T1 & $9(32.1)$ & $5(20.8)$ & 0.626 \\
\hline $\mathrm{T} 2$ & $4(14.3)$ & $3(12.5)$ & \\
\hline T3 & $15(53.6)$ & $16(66.7)$ & \\
\hline \multicolumn{4}{|l|}{ Lymph node metastasis } \\
\hline NO & $21(75.0)$ & $16(66.6)$ & 0.321 \\
\hline N1 & $6(21.4)$ & $4(16.7)$ & \\
\hline N2 & $1(3.6)$ & $4(16.7)$ & \\
\hline Lymphovascular invasion & $6(21.4)$ & $7(29.1)$ & 0.541 \\
\hline Perineural invasion & $3(10.7)$ & $3(12.5)$ & 1.000 \\
\hline \multicolumn{4}{|l|}{ TNM stage } \\
\hline I & $11(39.3)$ & $8(33.3)$ & 0.831 \\
\hline$\|$ & $10(35.7)$ & $8(33.3)$ & \\
\hline $\mid \|$ & 7 (25.0) & 8 (33.3) & \\
\hline
\end{tabular}

Values are presented as number (\%) or mean \pm standard deviation unless otherwise stated. S+1-LAR = single-incision plus one port laparoscopic low anterior resection; M-LAR = multi-port laparoscopic low anterior resection; $\mathrm{WD}=$ well-differentiated adenocarcinoma; $\mathrm{MD}=$ moderately differentiated adenocarcinoma; $\mathrm{PD}=$ poorly differentiated adenocarcinoma; $\mathrm{MUC}=$ mucinous adenocarcinoma. 
sion, perineural invasion, or histological TNM stage. All patients in both groups had negative distal and radial resection margins (Table 4).

\section{DISCUSSION}

Since its first description in 2008, many surgeons have reported their experiences in the single incision laparoscopic surgery for right colectomy for colorectal cancer. ${ }^{11}$ The procedure has yielded good postoperative outcomes in terms of complications, morbidity, mortality, and oncologic outcomes. ${ }^{1,8,9,12}$ Moreover, case-control or comparative studies have reported comparable surgical outcomes between single incision laparoscopic or single incision plus one-port laparoscopic and multi-port procedures. ${ }^{6,713,14} \mathrm{Liu}$ et al. reported the advantages associated with single incision laparoscopic surgery for sigmoid colon and rectal cancer surgery, such as shorter hospital stay, smaller incision length, accelerated return to normal bowel function, lower pain scores, and lower complication rates. ${ }^{12}$ The feasibility and benefits of S+1-LAR for rectosigmoid cancer, including reduction in operation time and postoperative pain have also been reported. ${ }^{10}$ Hirano et al. reported the short-term oncologic safety of S+1-LAR for rectal cancer. ${ }^{8}$ However, there are few published reports of studies comparing S+1-LAR with M-LAR for mid-to-low rectal cancer in relation to these outcome variables.

Rectal division in single incision laparoscopic low anterior resection is technically difficult and is associated with high levels of stress experienced by the surgeon because the shaft of the linear stapler is positioned parallel to the rectum when transecting the distal rectum. Dissection becomes more difficult as the transection line approaches the anal side. To overcome this technical limitation, we added an additional 12-mm port in the right lower abdominal quadrant for intraoperative endo-stapler insertion; the safety of this technique has been reported by several studies. $^{7-9}$

Postoperative pain can affect postoperative recovery, and less postoperative pain has been reported in association with single incision or single incision plus one port relative to multiport laparoscopic surgery, as fewer incisions may result in less pain. ${ }^{7,14}$ A randomized pilot study comparing postoperative pain in single incision and multi-port groups found that patients who underwent single incision laparoscopic operations reported significantly less pain at rest 6 hours after surgery and on postoperative days 1,3 , and 4 . $^{14}$ However, another study found no significant between-group differences in pain at rest or on postoperative days 1 and 2 during mobilization. The author analyzed these findings and determined that the routine use of patient-controlled opioid-based intravenous analgesia (PCIA) on postoperative days 1 and 2 may diminish the differences in pain intensity. ${ }^{7}$ Patients in our study reported significantly lower pain scores on postoperative day 1 , and there was no difference on postoperative days 3 and 7 with the routine use of PCIA in both groups until postoperative day 2. Because we encourage early mobilization and more than half of patients start ambulation on the first postoperative day in our hospital, pain associated with ambulation and motion may both have affected our results.

Port-site herniation is more common through umbilical ports than through other port sites. ${ }^{2}$ Incision length, wound infection, diabetes mellitus, and obesity are risk factors for port-site hernias. ${ }^{15}$ Some studies have reported that the single incision laparoscopic surgery through the umbilicus is associated with higher rates of port-site hernia than multi-port surgery, ${ }^{16}$ whereas other studies found no difference. ${ }^{17}$ In our study, there were two hernia patients in the S+1-LAR group and one in the M-LAR group, and this difference was not statistically significant. To our knowledge, there are no publications reporting large studies on trocar site hernias after the S+1-LAR for rectal cancer. Therefore, the S+1-LAR cannot yet be considered a risk factor of trocar site hernia in rectal cancer patients. Further large, long-term followup studies are needed. The frequencies of other postoperative complications were similar in both groups and were not associated with single-port techniques, as previously reported.

Katsuno G et al. reported good oncologic outcomes associated with single incision laparoscopic surgery, with a median followup period of 39 months and no significant difference in 5-year overall survival and disease free survival between single-port and multi-port surgery groups (100 vs. $95 \%, 97$ vs. $94 \%){ }^{13}$ Kim et al. also reported no significant difference in the 3-year OS and DFS between two similar groups ( 94.5 vs. $97.1 \%, 89.5$ vs. $87.4 \%$ ), with a median of 29.5 months of follow-up. ${ }^{6}$ Liu et al. found no difference in the 3-year DFS between the S+1-LAR and M-LAR groups (91.7 vs. $89.6 \%)^{7}$

We have several limitation in this study. Firstly, this study was retrospective design which could cause selection bias. Secondly, preoperative chemoradiation therapy rates were low in this study. Due to financial constraints and regional characteristics, patients could not take preoperative chemoradiation therapy despite the risk of local recurrence. Thirdly, there were heterogeneities in the S+1-LAR group in this study. Stoma site specimen extraction could increase the postoperative ileostomy complication rate including parastomal hernia. ${ }^{18}$ Fortunately, in our study, parastomal hernia did not occur in the group that used the ileostomy site for the main incision. However, these heterogeneities could have affected the surgical outcomes.

We demonstrated the short-term clinical safety of S+1-LAR for rectal cancer. This is a promising alternative laparoscopic method for mid-to-low rectal cancer. However, because of several limitations, large-scale randomized control studies with longer follow-up periods are needed to confirm the advantages of S+1LAR compared with M- LAR. 


\section{ORCID}

Jae Young Kwak, https://orcid.org/0000-0001-8263-2260

Kwan Mo Yang, https://orcid.org/0000-0001-9190-3296

Myeong Sik Han, https://orcid.org/0000-0001-6918-4432

\section{AUTHORS' CONTRIBUTIONS}

Conceptualization: Jae Young Kwak. Formal analysis: Jae Young Kwak. Methodology: Jae Young Kwak and Kwan Mo Yang. Writing-original draft: Jae Young Kwak and Myeong Sik Han. Writing-review and editing: Jae Young Kwak, Kwan Mo Yang, and Myeong Sik Han.

\section{CONFLICT OF INTEREST}

None.

\section{FUNDING}

None.

\section{ACKNOWLEDGMENTS}

None.

\section{REFERENCES}

1) Tei M, Otsuka M, Suzuki Y, Kishi K, Tanemura M, Akamatsu H. Safety and feasibility of single-port laparoscopic low anterior resection for upper rectal cancer. Am J Surg 2018;216:1101-1106.

2) Owens M, Barry M, Janjua AZ, Winter DC. A systematic review of laparoscopic port site hernias in gastrointestinal surgery. Surgeon 2011;9:218-224.

3) Poon JT, Cheung CW, Fan JK, Lo OS, Law WL. Single-incision versus conventional laparoscopic colectomy for colonic neoplasm: a randomized, controlled trial. Surg Endosc 2012;26:2729-2734.

4) Cianchi F, Staderini F, Badii B. Single-incision laparoscopic colorectal surgery for cancer: state of art. World J Gastroenterol 2014;20:60736080.

5) Li HJ, Huang L, Li TJ, Su J, Peng LR, Liu W. Short-Term Outcomes of Single-Incision Versus Conventional Laparoscopic Surgery for Colorectal Diseases: Meta-Analysis of Randomized and Prospective Evidence. J Gastrointest Surg 2017;21:1931-1945.

6) Kim CW, Cho MS, Baek SJ, et al. Oncologic outcomes of single-incision versus conventional laparoscopic anterior resection for sigmoid colon cancer: a propensity-score matching analysis. Ann Surg Oncol
2015;22:924-930.

7) Liu R, Wang Y, Zhang Z, et al. Assessment of treatment options for rectosigmoid cancer: single-incision plus one port laparoscopic surgery, single-incision laparoscopic surgery, and conventional laparoscopic surgery. Surg Endosc 2017;31:2437-2450.

8) Hirano Y, Hattori M, Douden K, Shimada M, Hashizume Y. ShortTerm Clinical and Oncological Outcomes after Single-Incision Plus One Port Laparoscopic Anterior Resection for Rectal Cancer. Dig Surg 2018;35:111-115.

9) Kawahara H, Misawa T, Watanabe K, et al. Feasibility of Singleincision Laparoscopic Surgery plus One Assist Port for Anterior Resection. Anticancer Res 2016;36:467-469.

10) Wang $Y$, Deng H, Mou T, et al. Short-term outcomes of singleincision plus one-port laparoscopic versus conventional laparoscopic surgery for rectosigmoid cancer: a randomized controlled trial. Surg Endosc 2019;33:840-848.

11) Remzi FH, Kirat HT, Kaouk JH, Geisler DP. Single-port laparoscopy in colorectal surgery. Colorectal Dis 2008;10:823-826.

12) Liu X, Li JB, Shi G, Guo R, Zhang R. Systematic review of single-incision versus conventional multiport laparoscopic surgery for sigmoid colon and rectal cancer. World J Surg Oncol 2018;16:220.

13) Katsuno G, Fukunaga M, Nagakari K, Yoshikawa S, Azuma D, Kohama S. Short-term and long-term outcomes of single-incision versus multi-incision laparoscopic resection for colorectal cancer: a propensity-score-matched analysis of 214 cases. Surg Endosc 2016;30:13171325.

14) Bulut $\mathrm{O}$, Aslak KK, Levic K, et al. A randomized pilot study on single-port versus conventional laparoscopic rectal surgery: effects on postoperative pain and the stress response to surgery. Tech Coloproctol 2015;19:11-22.

15) Comajuncosas J, Hermoso J, Gris P, et al. Risk factors for umbilical trocar site incisional hernia in laparoscopic cholecystectomy: a prospective 3-year follow-up study. Am J Surg 2014;207:1-6.

16) Antoniou SA, Morales-Conde S, Antoniou GA, Granderath FA, Berrevoet F, Muysoms FE. Single-incision laparoscopic surgery through the umbilicus is associated with a higher incidence of trocar-site hernia than conventional laparoscopy: a meta-analysis of randomized controlled trials. Hernia 2016;20:1-10.

17) Christoffersen MW, Brandt E, Oehlenschläger J, et al. No difference in incidence of port-site hernia and chronic pain after single-incision laparoscopic cholecystectomy versus conventional laparoscopic cholecystectomy: a nationwide prospective, matched cohort study. Surg Endosc 2015;29:3239-3245.

18) Li W, Benlice C, Stocchi L, Kessler H, Gorgun E, Costedio M. Does stoma site specimen extraction increase postoperative ileostomy complication rates? Surg Endosc 2017;31:3552-3558. 